\title{
FITOSSOCIOLOGIA E ESTRUTURA DIAMÉTRICA DA MATA DE GALERIA DO TAQUARA, NA RESERVA ECOLÓGICA DO IBGE, DF ${ }^{1}$
}

\author{
Manoel Cláudio da Silva Júnior²
}

\begin{abstract}
RESUMO - (Florística e estrutura na mata do Taquara, Reserva Ecológica do IBGE, DF). As matas de Galeria, apesar de legalmente protegidas, vêm sendo sistematicamente substituídas visando a outras finalidades. As árvores $\left(\mathrm{DAP}^{3} 5 \mathrm{~cm}\right.$ ) na mata de Galeria do córrego do Taquara, na Reserva Ecológica do IBGE, no Distrito Federal, foram amostradas pelo método de quadrantes, com 250 pontos, dispostos em linhas estabelecidas ao longo do comprimento do córrego, desde as margens até os limites entre a mata e o cerrado. A amostragem de 1.000 árvores resultou na composição florística, na fitossociologia e na distribuição dos diâmetros dos troncos. No total foram amostradas 110 espécies de 49 famílias. O índice de diversidade de Shannon \& Wiener foi estimado em 4,25 nats.ind ${ }^{-1}$. As famílias mais importantes foram: Leguminosae (lato sensu), Rubiaceae, Anacardiaceae, Euphorbiaceae e Sapindaceae, enquanto as espécies principais foram: Tapirira guianensis, Copaifera langsdorffii, Lamanonia ternata, Anadenanthera colubrina var. cebil, Piptocarpha macropoda, Alibertia macrophylla, Matayba guianensis, Pera glabrata, Guettarda viburnioides e Ixora warmingii. As estimativas da densidade e da área basal total foram de 1.573 árvores.ha-1 e 38,5 m².ha-1 respectivamente. A distribuição diamétrica indicou uma curva tendendo para o ' $\mathrm{J}$ ' invertido, evidenciando os baixos níveis de distúrbios ocorridos na mata. Uma comparação florística conduzida entre 21 matas de Galeria no Distrito Federal apontou a mata do Taquara como uma área rica, com 110 (29,1\%) das 378 espécies listadas e similaridade de Sørensen variando entre 0,34 e 0,80, nas matas incluídas.
\end{abstract}

Palavras-chave: Árvores, cerrado, hot spot da biodiversidade mundial, conservação.

\section{PHYTOSOCIOLOGY AND DIAMETRIC ESTRUCTURE OF TAQUARA GALLERY FOREST, AT IBGE ECOLOGICAL RESERVE, DF}

\begin{abstract}
Floristics and structure of the, IBGE, Federal District, Brazil). Despite been protected by law gallery forests have been systematically replaced by other uses. The study focused on the Taquara gallery forest within the Ecological Reserve of the "Instituto Brasileiro de Geografia e Estatística" in the Federal District, Central Brazil. One thousand trees $\left(D B H^{3} 5 \mathrm{~cm}\right)$ were recorded using the point centered-quarter (PCQ) method. A total of 250 sampling points were established in sampling lines from the stream margins to the forest-cerrado border and distributed from the head to the mouth of the stream. The floristic composition, phytosociology and diameter distribution were analyzed. One hundred and ten species of 49 families were found. The most important families were: Leguminosae, Rubiaceae, Anacardiaceae, Euphorbiaceae e Sapindaceae, while the most important species were: Tapirira guianensis, Copaifera langsdorffii, Lamanonia ternata, Anadenanthera colubrina var. cebil, Piptocarpha macropoda, Alibertia macrophylla, Matayba guianensis, Pera glabrata, Guettarda viburnioides e Ixora warmingii. Density and basal area were estimated as 1573 trees.ha ${ }^{-1}$ and 38.5 $\mathrm{m}^{2}$. ha ${ }^{-1}$ respectively. Diameter distribution indicated a tendency to an inverted ' $J$ ' curve revealing low levels of disturbance in this gallery forest. A floristic comparison with 21 gallery forests in the Federal District indicated Taquara gallery forest as a rich site including 110 (29,1\%) of the 378 species recorded and Sørensen similarities ranging from 0,34 to 0,80 within the studied forests.
\end{abstract}

Key words - Trees, cerrado, world biodiversity hot spot, conservation.

\footnotetext{
${ }^{1}$ Recebido para publicação em 16.6.2003 e aceito para publicação em 08.6.2004.

${ }^{2}$ Universidade de Brasília, Departamento de Engenharia Florestal, Caixa Postal 04357, 70919-970, Brasília, DF. <mcsj@unb.br>.
} 


\section{INTRODUÇÃO}

No Brasil, a composição florística de comunidades vegetais ainda é desconhecida em extensões consideráveis de seu território florestado. Estudos florísticos são básicos para a atualização das floras regional e nacional, pesquisa dos potenciais diversos das nossas plantas e para o entendimento de padrões de distribuição geográfica das espécies e de como esses padrões são influenciados pela latitude, longitude, altitude e por fatores ambientais como clima, solos (classes, gradientes, fertilidade e umidade). Atualmente, em razão do lamentável estado de conservação da maioria das formações vegetais brasileiras, esses estudos ganham importância maior. A florística serve ainda para programas de recuperação de áreas degradadas, em níveis local e regional, na ausência de estudos específicos de cada localidade (FELFILI et al., 2001ab; SILVAJÚNIOR et al., 2001).

As estimativas atuais para o Distrito Federal indicam que cerca de $60 \%$ da área original ocupada pelas matas de galeria já foi substituída para outros usos (UNESCO, 2003). As unidades de conservação, testemunhos das situações menos degradadas, vêm se tornando ilhas de vegetação limitadas em fluxo gênico e sujeitas aos efeitos de borda como plantas invasoras e aumento da freqüência de incêndios, entre tantos outros (OLIVEIRA-FILHO et al., 1997; KELMANN et al., 1998; FELFILI, 2000). Assim, as áreas estudadas servem para gerar conhecimentos necessários ao desenvolvimento de técnicas de manejo que melhorem o desempenho de suas finalidades, quais sejam a proteção dos mananciais hídricos dos solos, bem como da flora e da fauna associadas, conforme consta na Lei 7.511, de 07/07/1986 (SILVA JÚNIOR, 2001).

As matas de galeria formam comunidades florestais conspícuas em meio às outras comunidades campestres e savânicas típicas do Brasil central (RIBEIRO e WALTER, 1998). A ocorrência dessas matas está confinada aos fundos dos vales, com inclinações suaves ou acentuadas, que estão delimitadas com o campo limpo e menos freqüentemente com outras comunidades na região (FELFILI et al., 1994). Encaixadas nos fundos dos vales, as matas de galeria compõem gradientes ambientais que possibilitam a colonização por grande variedade de espécies e se tornam excelentes laboratórios naturais, onde as relações vegetação ambiente podem ser avaliadas (SILVA JÚNIOR, 1995,
1998). Felfili et al. (2001b) indicam a grande importância das matas de galeria para a diversidade fanerogâmica no bioma cerrado, uma vez que contribuem com $33 \%$ do número total de espécies, apesar de ocuparem área reduzida de cerca de 5\% em relação às demais fitofisionomias.

O Distrito Federal comporta nascentes de tributários de três grandes bacias hidrográficas do Brasil: do rio Amazonas, do rio São Francisco e do rio Paraná. Oliveira-Filho e Ratter (1995), estudando a origem das florestas no Brasil central, concluíram que as matas de galeria no Distrito Federal se assemelham floristicamente às florestas da bacia do rio Paraná, corroborando a sugestão anterior de Ab’Saber (1983).

Recentemente, a análise de 21 levantamentos florísticos realizados em matas de galerias associadas a córregos tributários da bacia dos rios AraguaiaTocantins, Paraná e São Francisco, evidenciaram que, independentemente da grande bacia hidrográfica, os níveis de fertilidade e umidade dos solos regem os grupos em níveis de maior similaridade nessas matas. Dentro de subgrupos, inundáveis ou não, a proximidade geográfica foi fator importante para a similaridade entre matas (SILVA JÚNIOR et al., 2001b).

O presente estudo foi conduzido na mata do Taquara, na Reserva do IBGE, e teve por objetivo contribuir para o conhecimento da florística, fitossociologia e estrutura diamétrica das matas de galeria do Brasil central.

\section{MATERIAL E MÉTODOS}

A Reserva Ecológica do IBGE (15 $56^{\prime} \mathrm{S}$ e $47^{\circ} 56^{\prime}$ W) ocupa 1.300 ha em altitudes variáveis, entre 1.048 e $1.160 \mathrm{~m}$. A área esteve protegida do fogo e de maiores distúrbios por cerca de 20 anos (PEREIRA et al., 1989, 1993). O IBGE, em conjunto com o Jardim Botânico de Brasília (JBB) e a Fazenda Água Limpa (FAL-UnB), soma aproximadamente 10.000 ha da área core de preservação da APA dos ribeirões do Gama e Cabeça-de-Veado e da Reserva da Biosfera do Cerrado (UNESCO, 2000).

Segundo a classificação de Köppen, o clima é do tipo Aw. Os dados da estação meteorológica do IBGE, no período de 1979 a 1985, indicam precipitação média anual de $1.436 \mathrm{~mm}$, temperaturas máximas e mínimas de $26,3^{\circ} \mathrm{C}$ e $15,8^{\circ} \mathrm{C}$, respectivamente, e temperatura média de $20,8^{\circ} \mathrm{C}$ (PEREIRA et al., 1989, 1993). Segun- 
do esses autores, nesse mesmo período a umidade do ar na época das chuvas variou entre 72 e $83 \%$ e no período seco, entre 58 e $67 \%$, com média anual de $73 \%$. A evapotranspiração média anual foi de 1771 mm, o que invariavelmente causa déficit hídrico.

Os Latossolos Vermelho e Vermelho-Amarelo cobrem cerca de $70 \%$ da superfície plana. Associados com as áreas dissecadas, encontram-se Cambissolos e Gleissolos e Plintossolos (EMBRAPA, 1999).

As comunidades vegetais associadas ao interflúvio somam $83,7 \%$ da reserva, enquanto a vegetação associada às áreas dissecadas, como o campo limpo e as matas de galeria, cobre a área restante, ou 16,3\% do total (PEREIRA et al., 1989, 1993).

O córrego do Taquara nasce na reserva e contribui para a microbacia dos córregos do Gama que deságua no Lago Paranoá, que compõe a bacia do rio São Bartolomeu, que flui para a bacia do rio Paraná.

As análises de 64 amostras superficiais $(0-20 \mathrm{~cm})$ dos solos na mata de galeria do Taquara resultaram no $\mathrm{pH}$ variando entre 4,7 e 6,4, com média de 5,3. Apenas $22 \%$ das amostras foram classificadas como muito ácidas e $10 \%$ como levemente ácidas, com $\mathrm{pH}>6,0$. Os altos níveis de matéria orgânica (MO) variaram entre 6,78 e 24,39\% ( $\chi=13,35)$. O valor médio de Al foi de 1,15 $\mathrm{cmol}_{\mathrm{c}} / \mathrm{kg}$, com teores elevados para $50 \%$ das amostras. A saturação de $\mathrm{Al}$ foi elevada para $80 \%$ das amostras, sendo $61 \%$ delas classificadas como álicas. Teores elevados de $\mathrm{Ca}+\mathrm{Mg}$ foram encontrados em $38,7 \%$ das amostras. O Ca, com valores médios de $5,92 \mathrm{cmol}_{c} / \mathrm{kg}$, contribuiu, em geral, com $71 \%$ do total de $\mathrm{Ca}+\mathrm{Mg}$ encontrados nas amostras. Esses resultados indicaram que os solos do Taquara contêm seis vezes mais Ca que os das matas do Monjolo e Pitoco. No K, a variação foi de 0,10 para $0,89 \mathrm{cmol}_{\mathrm{c}} / \mathrm{kg}$, nos solos do Taquara. Os níveis de $\mathrm{P}$ foram muito maiores que os valores médios observados nos solos do cerrado, variando entre 0,9 e 22,2 ( $\chi=4,04)$ (SILVA JÚNIOR, 1995).

No geral, os solos na mata do Taquara, em relação aos solos das matas do Monjolo e Pitoco, também na reserva do IBGE, apresentaram níveis mais altos de $\mathrm{pH}, \mathrm{MO}, \mathrm{Ca}, \mathrm{Mg}, \mathrm{K}, \mathrm{Zn}, \mathrm{Mn}$ e areia grossa, níveis intermediários de $\mathrm{P}$ e silte e níveis mais baixos de $\mathrm{Al}$, $\mathrm{H}+\mathrm{Al}$, Fe e $\mathrm{Cu}$, bem como saturação de $\mathrm{Al}$ e argila (SILVA JÚNIOR, 1995).
Árvores com DAP $\geq 5 \mathrm{~cm}$ foram amostradas com a aplicação de 250 pontos de amostragem pelo método de quadrantes (COTTAM e CURTIS, 1956), dispostos em linhas de amostragem alocadas em ambas as margens, ao longo de toda a extensão da mata, desde as margens do córrego até a borda com a vegetação do cerrado. A distância entre pontos e entre linhas de amostragem foi mantida em $10 \mathrm{~m}$.

No campo foram anotados os DAP e as distâncias dos indivíduos, incluindo-se o raio do tronco (ASHBY, 1972) até os pontos de amostragem. Todos esses pontos e árvores amostrados foram identificados com placas numeradas.

As coleções do material botânico foram depositadas nos herbários da Universidade de Brasília (UB), do IBGE (IBGE) e do Royal Botanic Garden em Edinburgo (E). O sistema de classificação adotado foi o de G.L. Stebbings (1974).

A curva do número de espécies em relação ao número de pontos de amostragem foi construída conforme Mueller-Dombois e Ellenberg (1974).

Os índices de Shannon (H') e de Pielou (eqüabilidade) (J') estimaram a diversidade, de acordo com Pielou (1975).

A distribuição do número de indivíduos em classes de diâmetro avaliou o ciclo de vida das espécies presentes. O quociente 'q' de Liocourt para avaliar o recrutamento foi calculado pela divisão do número de árvores em uma classe pelo número de árvores na classe anterior à mortalidade, obtida por 1-'q' (LIOCOURT, 1898, apud MEYER, 1952). O número de classes de diâmetro foi calculado de acordo com Spiegel (1976), para minimizar o número de classes sem representação.

A avaliação dos parâmetros fitossociológicos foi feita de acordo com Mueller-Dombois e Ellenberg (1974).

\section{RESULTADOS E DISCUSSÃO}

\subsection{A amostragem}

A metade do número de pontos aplicados (125) foi suficiente para amostrar $83 \%$ das 110 espécies encontradas (Figura 1). A inclusão de outros 125 pontos, ou mais 500 indivíduos resultou na adição de 19 (17\%) espécies.

\footnotetext{
R. Árvore, Viçosa-MG, v.28, n.3, p. 419-428, 2004
} 


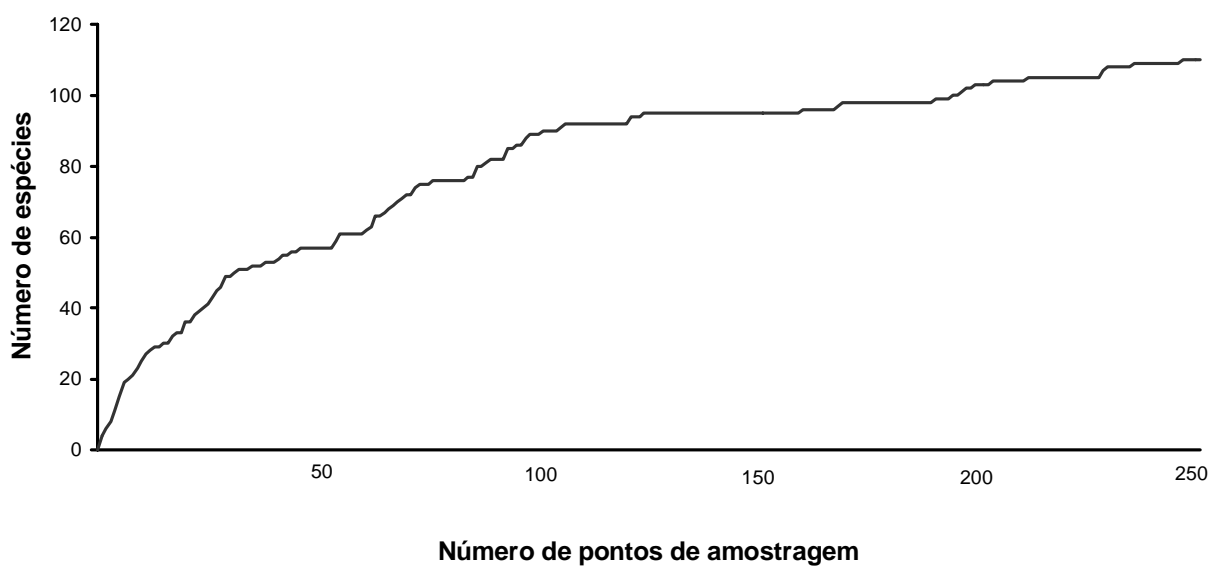

Figura 1 - Curva do número de pontos de amostragem (250) versus o número de espécies amostradas (110) na mata do Taquara na Reserva Ecológica do IBGE, em Brasília, DF.

Figure 1 - Curve of number of sampling points (250) versus number pf species sampled (110) in Taquara forest, Brasília, $D F$.

Nas matas de galeria, em geral, espera-se a estabilização tardia da curva devido à grande heterogeneidade florística, como conseqüência dos gradientes ambientais, impostos pela inclinação do terreno e suas conseqüências na umidade e fertilidade dos solos (FELFILI, 1993, 1998; OLIVEIRA-FILHO et al., 1994; SILVA JÚNIOR, 1995, 1997, 1998; SILVA JÚNIOR et al., 1996; HARIDASAN et al., 1997; REZENDE et al., 1997; WALTER e RIBEIRO, 1997; NÓBREGA, 1999).

A curva obtida indica fase tardia de sucessão, com baixos níveis de distúrbios na mata do Taquara, onde há grande diferenciação de nichos (YODZIS, 1978, citado por LEPS e STURSA, 1989).

\subsection{A diversidade florística}

Os 1.000 indivíduos amostrados foram distribuídos em 110 espécies. O índice de equabilidade de Pielou foi de $90 \%$ e o índice de diversidade de Shannon e Weiner, de 4,25 nats. ind ${ }^{-1}$, o maior verificado em matas de galeria no Brasil central, variando entre 2,51 e 4,25 nats. ind ${ }^{-1}$ (SILVA JÚNIOR et al., 1998, 2001).

\subsection{As famílias}

No total foram amostradas 48 famílias, dentre as quais se destacaram as cinco mais ricas (R), com as maiores densidades por hectare (D), as maiores áreas basais por hectare (AB) e os mais altos valores de importância (IVI): Anacardiaceae (D, AB, IVI), Burseraceae (IVI), Cunoniaceae (AB), Euphorbiaceae (R, $\mathrm{D}, \mathrm{AB}, \mathrm{IVI})$, Lauraceae (R, D, AB, IVI), Leguminosae (R, D, AB, IVI), Moraceae (R, D), Myrtaceae (R, D), Rubiaceae (R, D, AB, IVI), Sapindaceae (D, AB) e Vochysiaceae (AB, IVI).

A comparação entre 21 matas de galeria no DF (SILVA JÚNIOR et al., 2001) incluiu Anacardiaceae, Annonaceae, Apocynaceae, Combretaceae, Dichapetalaceae, Euphorbiaceae, Hippocrateaceae, Icacinaceae, Lauraceae, Leguminosae, Melastomataceae, Moraceae, Myristicaceae, Myrsinaceae, Myrtaceae, Rubiaceae, Sapindaceae e Sapotaceae, no grupo das famílias comuns às matas do Distrito Federal, por terem ocorrido em 18 das 21 localidades. Dentre essas, Anacardiaceae, Annonaceae, Leguminosae e Rubiaceae ocorreram nas 21 áreas e foram consideradas abundantes.

\subsection{As espécies}

Foram amostradas 110 espécies pertencentes a 91 gêneros (Tabela 1). A distância média entre árvores foi de $2,51 \mathrm{~m}$, que resultou na densidade total de 1.573 árvores.ha- ${ }^{-1}$ e na área basal total de $38,5 \mathrm{~m}^{2}$.ha ${ }^{-1}$.

Silva Júnior et al., (2001) listaram 378 espécies arbóreas em 21 matas de galeria no Distrito Federal; as 110 espécies na mata do Taquara contribuiram com 29,1\% desse total. A similaridade florística (Sørensen) 
entre a mata do Taquara e as outras 20 do Distrito Federal variou de 0,34 em relação à mata Cemave do Parque Nacional de Brasília a 0,80 com a mata do Pitoco do IBGE. Nove das 20 comparações resultaram em índices menores que 50\%. A presença de aproximadamente 30\% das espécies de matas de galeria do Distrito Federal, no Taquara, aliada à baixa similaridade florística entre as matas, ressalta a importância da proteção da mata do Taquara no IBGE.

\subsection{A distribuição dos diâmetros}

Foram estimadas 1.573 árvores.ha $^{-1}$, que distribuídos em 16 classes de diâmetro (Figura 2). Na primeira classe de DAP (5-<9,9 cm) ocorreram 52\% das árvores amostradas, e $91 \%$ apresentaram DAP menor que 30 $\mathrm{cm}$. A maior árvore registrada foi Lamanonia ternata, com 89,9 cm de DAP. Somente oito das espécies amostradas: Anadenanthera colubrina var. cebil,

Tabela 1- Parâmetros fitossociológicos das árvores amostradas na mata de galeria do Taquara na Reserva Ecológica do IBGE, Brasília, DF (em que: N=número de árvores, DA=densidade absoluta, DR=densidade relativa, ABA=área basal absoluta, $\mathrm{ABR}=$ área basal relativa e $\mathrm{FR}=$ freqüência relativa e IVI=índice do valor de importância)

Table 1 - Phytosociological parameters of trees sampled in Taquara gallery forest, IBGE Ecological Reserve, Brasília, $D F(N=$ tree number, $D A=$ absolute density, $D R=$ relative density, $A B A=$ absolute basal area, $A B R=$ relative basal area, $F R=$ relative frequency, $I V I=$ importance value index)

\begin{tabular}{|c|c|c|c|c|c|c|c|c|c|}
\hline & \multirow[t]{2}{*}{ Espécies } & \multirow[t]{2}{*}{ Famílias } & \multirow[t]{2}{*}{$\mathrm{N}$} & \multirow{2}{*}{$\frac{\text { DA }}{\text { n/ha }}$} & \multirow{2}{*}{$\begin{array}{c}\mathrm{DR} \\
\%\end{array}$} & \multirow{2}{*}{$\frac{\mathrm{ABA}}{\mathrm{cm} 2 / \mathrm{h} \mathrm{a}}$} & \multirow{2}{*}{$\frac{\mathrm{ABR}}{\%}$} & \multirow{2}{*}{$\begin{array}{l}\text { FR } \\
\%\end{array}$} & \multirow[t]{2}{*}{ IVI } \\
\hline & & & & & & & & & \\
\hline$\overline{1}$ & Tapirira guianensis Aublet & Anacardiaceae & 70 & 111,7 & 7,00 & 20099 & 4,35 & 5,72 & 17,07 \\
\hline & Mortas & & 48 & 73,9 & 4,80 & 31572 & 6,85 & 4,96 & 16,62 \\
\hline 2 & Copaifera langsdorffii Desf. & Leg.-Caesalpinioideae & 30 & 47,2 & 3,00 & 33170 & 7,28 & 3,13 & 13,41 \\
\hline 3 & Lamanonia ternata Vell. & Cunoniaceae & 33 & 25,2 & 3,30 & 28623 & 4,45 & 3,24 & 10,98 \\
\hline 4 & $\begin{array}{l}\text { Anadenanthera colubrina var. } \\
\text { cebil (Griseb.) Altschul }\end{array}$ & Leg.-Mimosoideae & 42 & 33,0 & 4,20 & 22110 & 2,76 & 3,78 & 10,74 \\
\hline 5 & Piptocarpha macropoda Baker. & Compositae & 36 & 55,1 & 3,60 & 12936 & 3,13 & 3,13 & 9,85 \\
\hline 6 & Alibertia macrophylla Schum. & Rubiaceae & 23 & 64,5 & 2,30 & 4872 & 3,31 & 2,37 & 7,98 \\
\hline 7 & Matayba guianensis Aublet. & Sapindaceae & 19 & 51,9 & 1,90 & 6018 & 3,84 & 1,94 & 7,68 \\
\hline 8 & Pera glabrata Poepp. ex Baill. & Euphorbiaceae & 16 & 31,5 & 1,60 & 12373 & 4,53 & 1,51 & 7,64 \\
\hline 9 & $\begin{array}{l}\text { Guettarda viburnioides } \\
\text { Cham. \& Schlecht. }\end{array}$ & Rubiaceae & 20 & 47,2 & 2,00 & 4604 & 3,21 & 2,16 & 7,37 \\
\hline 10 & Ixora warmingii Muell. Arg. & Rubiaceae & 30 & 36,2 & 3,00 & 7324 & 1,20 & 2,91 & 7,11 \\
\hline 11 & Platypodium elegans Vog. & Leg.-Caesalpinioideae & 22 & 28,3 & 2,20 & 9972 & 2,74 & 1,94 & 6,88 \\
\hline 12 & Protium almecega Marchand & Burseraceae & 19 & 34,6 & 1,90 & 7996 & 2,10 & 1,83 & 5,83 \\
\hline 13 & $\begin{array}{l}\text { Sclerolobium paniculatum var. I } \\
\text { rubiginosum (Mart. ex Tul.) Bent }\end{array}$ & $\begin{array}{l}\text { Leg.-Caesalpinioideae } \\
\text { th. }\end{array}$ & 18 & 29,9 & 1,80 & 8132 & 2,02 & 1,83 & 5,66 \\
\hline 14 & $\begin{array}{l}\text { Tapura amazonica } \\
\text { Poepp. \& Endl. }\end{array}$ & Dichapetalaceae & 18 & 28,3 & 1,80 & 5870 & 1,57 & 1,83 & 5,20 \\
\hline 15 & Emmotum nitens Miers. & Icacinaceae & 13 & 20,5 & 1,30 & 8425 & 2,24 & 1,29 & 4,83 \\
\hline 16 & Diospyros hispida A. DC. & Ebenaceae & 17 & 26,7 & 1,70 & 4858 & 1,26 & 1,73 & 4,69 \\
\hline 17 & Hymenaea stilbocarpa Hayne & Leg.-Caesalpinioideae & 19 & 9,4 & 1,90 & 12842 & 0,40 & 1,83 & 4,13 \\
\hline 18 & $\begin{array}{l}\text { Pseudobombax tomentosum } \\
\text { (Mart. \& Zucc.) A. Robyns }\end{array}$ & Bombacaceae & 15 & 11,0 & 1,50 & 11243 & 0,93 & 1,51 & 3,94 \\
\hline 19 & Myrcia rostrata DC. & Myrta & 6 & 29,9 & 0,60 & 1367 & 2,68 & 0,65 & 3,92 \\
\hline 20 & Byrsonima laxiflora Griseb. & Malpighiaceae & 7 & 22,0 & 0,70 & 4305 & 2,44 & 0,76 & 3,90 \\
\hline 21 & $\begin{array}{l}\text { Cheiloclinium cognatum } \\
\text { (Miers) A. C. Smith }\end{array}$ & Hippocrateaceae & 16 & 25,2 & 1,60 & 2209 & 0,57 & 1,62 & 3,79 \\
\hline 22 & $\begin{array}{l}\text { Guapira graciliflora } \\
\text { (Mart. ex Schmidt) Lundell }\end{array}$ & Nyctaginaceae & 15 & 23,6 & 1,50 & 2818 & 0,75 & 1,51 & 3,76 \\
\hline 23 & Maprounea guianensis Aublet. & Euph & 15 & 23,6 & 1,50 & 2610 & 0,69 & 1,51 & 3,70 \\
\hline 24 & Ocotea corymbosa Mez. & Laur & 14 & 18,9 & 1,40 & 4292 & 0,86 & 1,40 & 3,67 \\
\hline 25 & Cupania vernalis Camb. & Sapindaceae & 12 & 23,6 & 1,20 & 2591 & 1,14 & 1,29 & 3,64 \\
\hline 26 & Myrcia tomentosa (Aublet) DC. & Myrtaceae & 15 & 20,5 & 1,50 & 3487 & 0,67 & 1,40 & 3,58 \\
\hline 27 & Symplocos nitens (Pohl) Benth. & Symplocaceae & 13 & 23,6 & 1,30 & 1729 & 0,91 & 1,29 & 3,50 \\
\hline 28 & Inga alba Wild. & Leg.-Mimosoideae & 13 & 20,5 & 1,30 & 2813 & 0,77 & 1,40 & 3,47 \\
\hline 29 & $\begin{array}{l}\text { Aspidosperma spruceanum } \\
\text { Benth. ex Muell. Arg. }\end{array}$ & Apocynaceae & 15 & 11,0 & 1,50 & 7522 & 0,45 & 1,51 & 3,46 \\
\hline 30 & $\begin{array}{l}\text { Schefflera morototoni (Aublet) } \\
\text { D. Frodin }\end{array}$ & Araliaceae & 7 & 22,0 & 0,70 & 1617 & 1,95 & 0,76 & 3,41 \\
\hline 31 & $\begin{array}{l}\text { Cousarea hydrangeifolia } \\
\text { Benth. \& Hook. }\end{array}$ & Rubiaceae & 12 & 22,0 & 1,20 & 1151 & 0,73 & 1,19 & 3,12 \\
\hline 32 & Qualea dichotoma (Mart.) Warm. & Vochysiaceae & 14 & 9,4 & 1,40 & 6670 & 0,30 & 1,40 & 3,10 \\
\hline
\end{tabular}


Tabela 1, cont.

Table 2, cont..

\begin{tabular}{|c|c|c|c|c|c|c|c|c|c|}
\hline & \multirow[t]{2}{*}{ Espécies } & \multirow[t]{2}{*}{ Famílias } & \multirow[t]{2}{*}{$\mathrm{N}$} & \multirow{2}{*}{$\frac{\mathrm{DA}}{\mathrm{n} / \mathrm{ha}}$} & \multirow{2}{*}{$\frac{\mathrm{DR}}{\%}$} & \multirow{2}{*}{$\frac{\mathrm{ABA}}{\mathrm{cm} 2 / \mathrm{h} \mathrm{a}}$} & \multirow{2}{*}{$\frac{\mathrm{ABR}}{\%}$} & \multirow{2}{*}{$\frac{\mathrm{FR}}{\%}$} & \multirow[t]{2}{*}{ IVI } \\
\hline & & & & & & & & & \\
\hline 33 & Jacaranda puberula Cham. & Bignoniaceae & 12 & 20,5 & 1,20 & 1447 & 0,46 & 1,29 & 2,95 \\
\hline 34 & Ocotea spixiana Mez & Lauraceae & 10 & 15,7 & 1,00 & 3370 & 0,87 & 1,08 & 2,95 \\
\hline 35 & Styrax guianensis A.DC. & Styracaceae & 9 & 18,9 & 0,90 & 1756 & 1,04 & 0,97 & 2,91 \\
\hline 36 & Aspidosperma subincanum Mart. & Apocynaceae & 11 & 14,2 & 1,10 & 4044 & 0,40 & 1,19 & 2,69 \\
\hline 37 & Symplocos mosenii Brand. & Symplocaceae & 5 & 17,3 & 0,50 & 2138 & 1,58 & 0,54 & 2,62 \\
\hline 38 & Aspidosperma discolor A. DC. & Apocynaceae & 8 & 12,6 & 0,80 & 3280 & 0,93 & 0,86 & 2,60 \\
\hline 39 & Callisthene major Mart. & Vochysiaceae & 7 & 7,9 & 0,70 & 5666 & 1,21 & 0,65 & 2,56 \\
\hline 40 & Terminalia glabrescens Mart. & Combretaceae & 5 & 12,6 & 0,50 & 3098 & 1,47 & 0,54 & 2,51 \\
\hline 41 & Machaerium acutifolium Vog. & Leg.-Faboideae & 9 & 14,2 & 0,90 & 2300 & 0,57 & 0,86 & 2,33 \\
\hline 42 & Xylopia sericea A. St. Hil. & Annonaceae & 8 & 14,2 & 0,80 & 1673 & 0,63 & 0,86 & 2,30 \\
\hline 43 & Hirtella glandulosa Spreng. & Chrysobalanaceae & 7 & 12,6 & 0,70 & 2442 & 0,84 & 0,76 & 2,29 \\
\hline 44 & Miconia cuspidata Naudin & Melastomataceae & 10 & 11,0 & 1,00 & 3629 & 0,27 & 0,97 & 2,25 \\
\hline 45 & Roupala brasiliensis Klotzch. & Proteaceae & 8 & 15,7 & 0,80 & 1058 & 0,42 & 0,86 & 2,08 \\
\hline 46 & Virola sebifera Aublet & Myristicaceae & 8 & 12,6 & 0,80 & 1604 & 0,42 & 0,86 & 2,08 \\
\hline 47 & Licania apetala (E.Mey.) Fritsch C & Chrysobalanaceae & 5 & 11,0 & 0,50 & 2068 & 1,01 & 0,54 & 2,05 \\
\hline 48 & Lafoensia pacari St. Hil. & Lythraceae & 7 & 9,4 & 0,70 & 2696 & 0,54 & 0,76 & 1,99 \\
\hline 49 & Cecropia lyratiloba Miq. & Moraceae & 2 & 11,0 & 0,20 & 1892 & 1,54 & 0,22 & 1,96 \\
\hline 50 & Hieronyma ferruginea Tul. & Euphorbiaceae & 6 & 9,4 & 0,60 & 2465 & 0,70 & 0,65 & 1,95 \\
\hline 51 & Margaritaria nobilis Linn. f. & Lauraceae & 7 & 7,9 & 0,70 & 3088 & 0,47 & 0,76 & 1,92 \\
\hline 52 & Siphoneugena densiflora Berg. & Myrtaceae & 6 & 6,3 & 0,60 & 3617 & 0,64 & 0,65 & 1,89 \\
\hline 53 & Vochysia tucanorum Mart. & Vochysiaceae & 5 & 11,0 & 0,50 & 1142 & 0,80 & 0,54 & 1,84 \\
\hline 54 & Nectandra cissiflora Nees & Lauraceae & 4 & 9,4 & 0,40 & 1747 & 0,94 & 0,43 & 1,77 \\
\hline 55 & Cecropia pachystachya Tréc. & Moraceae & 7 & 11,0 & 0,70 & 745 & 0,30 & 0,76 & 1,75 \\
\hline 56 & Piptadenia gonoacantha Macbr. & Leg.-Mimosoideae & 6 & 7,9 & 0,60 & 2292 & 0,45 & 0,65 & 1,70 \\
\hline 57 & $\begin{array}{l}\text { Eriotheca gracilipes } \\
\text { (K. Schum.) A. Robyns }\end{array}$ & Bombacaceae & 5 & 6,3 & 0,50 & 2948 & 0,61 & 0,54 & 1,65 \\
\hline 58 & Myrsine coriacea R.Br. & Myrsinaceae & 3 & 11,0 & 0,30 & 429 & 1,01 & 0,32 & 1,63 \\
\hline 59 & Tabebuia impetiginosa (Mart.) & andleyBignoniaceae & 4 & 7,9 & 0,40 & 1484 & 0,77 & 0,43 & 1,60 \\
\hline 60 & Micropholis rigida Pierre & Sapotaceae & 7 & 9,4 & 0,70 & 557 & 0,13 & 0,76 & 1,58 \\
\hline 61 & Guatteria sellowiana Schlecht. & Annonaceae & 3 & 9,4 & 0,30 & 331 & 0,85 & 0,32 & 1,47 \\
\hline 62 & Bauhinia rufa Steud. & eg.-Caesalpinioideae & 7 & 9,4 & 0,70 & 316 & 0,11 & 0,65 & 1,46 \\
\hline 63 & Guarea guidonia (L.) Sleumer & Meliaceae & 5 & 7,9 & 0,50 & 681 & 0,39 & 0,54 & 1,42 \\
\hline 64 & Ouratea castaneaefolia Engler & Ochnaceae & 5 & 7,9 & 0,50 & 400 & 0,38 & 0,54 & 1,42 \\
\hline 65 & Cordia sellowiana Cham. & Boraginaceae & 6 & 7,9 & 0,60 & 313 & 0,14 & 0,65 & 1,39 \\
\hline 66 & Tabebuia umbellata (Sond.) Sandw. & 7. Bignoniaceae & 6 & 7,9 & 0,60 & 1129 & 0,09 & 0,65 & 1,33 \\
\hline 67 & Eriotheca pubescens Schott. \& Endl. & dl. Bombacaceae & 6 & 6,3 & 0,60 & 1071 & 0,07 & 0,65 & 1,31 \\
\hline 68 & Euplassa inaequalis (Pohl) Engler & Proteaceae & 5 & 1,6 & 0,50 & 3201 & 0,11 & 0,54 & 1,15 \\
\hline 69 & Myrsine umbellata Mart. & Myrsinaceae & 5 & 6,3 & 0,50 & 527 & 0,10 & 0,54 & 1,14 \\
\hline 70 & Pseudolmedia guaranitica Hassl. & Moraceae & 5 & 6,3 & 0,50 & 501 & 0,29 & 0,32 & 1,12 \\
\hline 71 & Cardiopetalum calophyllum Schlecht & ht. Annonaceae & 4 & 6,3 & 0,40 & 467 & 0,26 & 0,43 & 1,09 \\
\hline 72 & Qualea multiflora Mart. & Vochysiaceae & 4 & 3,2 & 0,40 & 1815 & 0,22 & 0,43 & 1,05 \\
\hline 73 & Cryptocarya aschersoniana Mez & Lauraceae & 4 & 4,7 & 0,40 & 933 & 0,14 & 0,43 & 0,97 \\
\hline 74 & Gomidesia lindeniana $\mathrm{O}$. Berg & Myrtaceae & 3 & 4,7 & 0,30 & 849 & 0,24 & 0,32 & 0,87 \\
\hline 75 & Erythroxylum sp. & Erythroxylaceae & 3 & 4,7 & 0,30 & 793 & 0,22 & 0,32 & 0,84 \\
\hline 76 & Faramea cyanea Muell. Arg. & Rubiaceae & 2 & 4,7 & 0,20 & 727 & 0,42 & 0,22 & 0,83 \\
\hline 77 & Kielmeyera coriacea Mart. & Guttiferae & 3 & 4,7 & 0,30 & 589 & 0,21 & 0,32 & 0,83 \\
\hline 78 & Vitex polygama Cham. & Verbenaceae & 3 & 4,7 & 0,30 & 330 & 0,15 & 0,32 & 0,78 \\
\hline 79 & Maytenus salicifolia Reiss. & Celastraceae & 3 & 4,7 & 0,30 & 287 & 0,09 & 0,32 & 0,71 \\
\hline 80 & Prunus brasiliensis (Spreng.) Schott. & Rosaceae & 3 & 4,7 & 0,30 & 154 & 0,07 & 0,32 & 0,70 \\
\hline 81 & Pouteria ramiflora Radlk. & Sapotaceae & 3 & 4,7 & 0,30 & 152 & 0,04 & 0,32 & 0,66 \\
\hline 82 & Calophyllum brasiliense Cambess. & Guttiferae & 3 & 1,6 & 0,30 & 1445 & 0,04 & 0,32 & 0,66 \\
\hline 83 & $\begin{array}{l}\text { Pseudobombax longiflorum } \\
\text { (A. Robyns) A. Robyns }\end{array}$ & Bombacaceae & 2 & 3,2 & 0,20 & 605 & 0,16 & 0,22 & 0,57 \\
\hline 84 & $\begin{array}{l}\text { Myrciaria glanduliflora } \\
\text { (Kiaresk.) Mattos \& Legrand }\end{array}$ & Myrtaceae & 2 & 3,2 & 0,20 & 268 & 0,07 & 0,22 & 0,49 \\
\hline 85 & Alibertia edulis (L.C. Rich) A. Rich. & ch. Rubiaceae & 2 & 3,2 & 0,20 & 194 & 0,05 & 0,22 & 0,47 \\
\hline 86 & Luehea grandiflora Mart. \& Zucc. & Tiliaceae & 2 & 3,2 & 0,20 & 152 & 0,04 & 0,22 & 0,46 \\
\hline 87 & Cordia trichotoma Vell. ex Steud. & Boraginaceae & 2 & 3,2 & 0,20 & 136 & 0,04 & 0,22 & 0,45 \\
\hline 88 & Casearia grandiflora Cambess. & Flacourtiaceae & 2 & 3,2 & 0,20 & 130 & 0,03 & 0,22 & 0,45 \\
\hline
\end{tabular}


Tabela 1, cont.

Table 2, cont..

\begin{tabular}{|c|c|c|c|c|c|c|c|c|c|}
\hline & \multirow[t]{2}{*}{ Espécies } & \multirow[t]{2}{*}{ Famílias } & \multirow[t]{2}{*}{$\mathrm{N}$} & \multirow{2}{*}{$\frac{\mathrm{DA}}{\mathrm{n} / \mathrm{ha}}$} & \multirow{2}{*}{$\frac{\mathrm{DR}}{\%}$} & \multirow{2}{*}{$\frac{\mathrm{ABA}}{\mathrm{cm} 2 / \mathrm{h} \mathrm{a}}$} & \multirow{2}{*}{$\begin{array}{c}\text { ABR } \\
\%\end{array}$} & \multirow{2}{*}{$\frac{\mathrm{FR}}{\%}$} & \multirow[t]{2}{*}{ IVI } \\
\hline & & & & & & & & & \\
\hline 89 & Siparuna guianensis Aublet & Monimiaceae & 2 & 3,2 & 0,20 & 95 & 0,02 & 0,22 & 0,44 \\
\hline 90 & $\begin{array}{l}\text { Cybianthus gardnerii } \\
\text { (A. DC.) Agostini }\end{array}$ & Myrsinaceae & 2 & 3,2 & 0,20 & 81 & 0,02 & 0,22 & 0,43 \\
\hline 91 & Xylopia emarginata Mart. & Annonaceae & 1 & 3,2 & 0,10 & 64 & 0,19 & 0,11 & 0,40 \\
\hline 92 & Ocotea aciphylla (Nees) Mez & Lauraceae & 1 & 1,6 & 0,10 & 730 & 0,10 & 0,11 & 0,31 \\
\hline 93 & $\begin{array}{l}\text { Tabebuia serratifolia } \\
\text { (Vahl.) Nicholson }\end{array}$ & Bignoniaceae & 1 & 1,6 & 0,10 & 396 & 0,09 & 0,11 & 0,30 \\
\hline 94 & Chomelia pohliana Muell. Arg. & Rubiaceae & 1 & 1,6 & 0,10 & 336 & 0,09 & 0,11 & 0,30 \\
\hline 95 & $\begin{array}{l}\text { Blepharocalyx salicifolius } \\
\text { (Kunth) Berg }\end{array}$ & Myrtaceae & 1 & 1,6 & 0,10 & 336 & 0,06 & 0,11 & 0,27 \\
\hline 96 & Sacoglottis guianensis Benth. & Humiriaceae & 1 & 1,6 & 0,10 & 256 & 0,06 & 0,11 & 0,27 \\
\hline 97 & Tetragastris balsamifera Kuntze & Burseraceae & 1 & 1,6 & 0,10 & 249 & 0,04 & 0,11 & 0,24 \\
\hline 98 & Virola urbaniana Warb. & Myristicaceae & 1 & 1,6 & 0,10 & 242 & 0,03 & 0,11 & 0,24 \\
\hline 99 & $\begin{array}{l}\text { Hirtella gracilipes } \\
\text { (Hook. f.) Prance }\end{array}$ & Chrysobalanaceae & 1 & 1,6 & 0,10 & 136 & 0,03 & 0,11 & 0,24 \\
\hline 100 & Sloanea guianensis Benth. & Elaeocarpaceae & 1 & 1,6 & 0,10 & 112 & 0,02 & 0,11 & 0,23 \\
\hline 101 & Zanthoxylum rhoifolium Lam. & Rutaceae & 1 & 1,6 & 0,10 & 107 & 0,02 & 0,11 & 0,23 \\
\hline 102 & Mollinedia oligantha Perkins. & Monimiaceae & 1 & 1,6 & 0,10 & 85 & 0,02 & 0,11 & 0,22 \\
\hline 103 & Vismia guianesis (Aubl.) Choisy & Guttiferae & 1 & 1,6 & 0,10 & 66 & 0,01 & 0,11 & 0,22 \\
\hline 104 & Laplacea fruticosa (Schrad.) Kobuski & Theaceae & 1 & 1,6 & 0,10 & 51 & 0,01 & 0,11 & 0,22 \\
\hline 105 & Sorocea guilleminiana Gaud. & Moraceae & 1 & 1,6 & 0,10 & 51 & 0,01 & 0,11 & 0,22 \\
\hline 106 & Dalbergia foliolosa Benth. & Leg.-Faboideae & 1 & 1,6 & 0,10 & 51 & 0,01 & 0,11 & 0,22 \\
\hline 107 & Hedyosmum brasiliense Mart. & Chloranthaceae & 1 & 1,6 & 0,10 & 40 & 0,01 & 0,11 & 0,22 \\
\hline 108 & Ficus citrifolia P. Miller & Moraceae & 1 & 1,6 & 0,10 & 37 & 0,01 & 0,11 & 0,22 \\
\hline 109 & Miconia chartacea Triana & Melastomataceae & 1 & 1,6 & 0,10 & 36 & 0,01 & 0,11 & 0,22 \\
\hline 110 & Richeria obovata Pax \& K. Hoffm. & Euphorbiaceae & 1 & 1,6 & 0,10 & 32 & 0,01 & 0,11 & 0,22 \\
\hline Tot. & 110 espécies & 48 famílias & 1000 & 1573 & $100 \%$ & 384980 & $100 \%$ & $100 \%$ & $300 \%$ \\
\hline
\end{tabular}

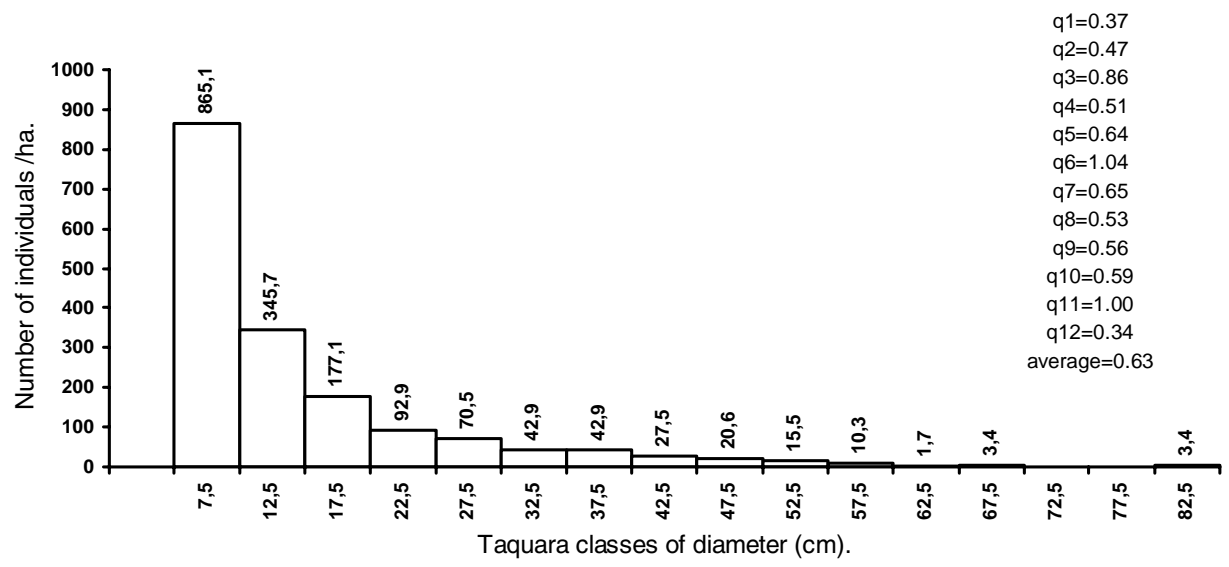

Figura 2 - Distribuição dos diâmetros e quocientes 'q' de Liocourt para a amostragem de 1.000 indivíduos na Mata de Galeria do Monjolo na RECOR, Brasília, DF.

Figure 2 - Distribuition of diameter and liocourt's ' $q$ ' quotients for the sampling of 1.000 individuals in Monjolo gallery forest, RECOR, Brasília, DF.

Copaifera langsdorffii, Euplassa inaequalis, Hymenaea coubaril var. stilbocarpa, Lamanonia ternata, Miconia cuspidata, Protium almecega e Pseudobombax tomentosum, apresentaram diâmetros maiores que
$50 \mathrm{~cm}$. As árvores amostradas em matas de galeria no Brasil central raramente excedem os 100 cm de diâmetro (FELFILI, 1997; SILVAJÚNIOR, 1995). Nas matas do Pitoco e Monjolo, no IBGE, as maiores árvores 
encontradas foram indivíduos de Copaifera langsdorffii, respectivamente com DAPs de 66,8 e 84,3 cm (SILVA JÚNIOR, 1995, 1999).

A baixa taxa de recrutamento entre classes foi apontada pelo quociente médio de Liocourt, calculado em 0,63. A sobrevivência nas menores classes foi abaixo da média calculada (Figura 2), indicando alta mortalidade natural.

A estrutura diamétrica revela a comunidade arbórea, no Taquara, composta principalmente por árvores pequenas. Felfili (1997) encontrou na mata do Gama (FAL-UnB), vizinha ao IBGE, 90\% das árvores com DAP menor que $45 \mathrm{~cm}$ e diâmetro máximo de cerca de 100 $\mathrm{cm}$.

O padrão da curva em ' J' invertido, com 47,1\% das árvores na primeira classe de diâmetro (5-9,9 cm), indica o balanço positivo entre recrutamento e mortalidade e caracteriza a mata do Taquara como autoregenerante. As variações no quociente 'q' (Figura 2) indicam taxas de recrutamento e mortalidade variáveis e a tendência de distribuição balanceada. Harper (1990) comentou que a maioria das florestas naturais apresenta distribuição dos diâmetros se aproximando do 'J' invertido.

Neste trabalho, destaca-se a mata de galeria do Taquara como possuidora de expressiva riqueza, com 110 espécies arbóreas, ressaltando sua relevância no cenário da conservação, por contar com cerca de 30\% da flora arbórea registrada nessa comunidade, no Distrito Federal.

\section{REFERÊNCIAS BIBLIOGRÁFICAS}

AB'SABER, A.N. O domínio dos cerrados: introdução ao conhecimento. Fundação Centro de Formação do Servidor Público, v. 3, n. 4, p. 41-55, 1983.

ASHBY, W.C. Distance measurements in vegetation study. Ecology, v. 53, p. 980-981, 1972.

COTTAM, G.; CURTIS, J.T. The use of distance measurements in phytosociological sampling. Ecology, v. 37 p. 451-460, 1956.
Empresa Brasileira de Pesquisa Agropecuária EMBRAPA. Sistema brasileiro de classificação de solos. Brasília: Embrapa Produção de Informação; Rio de Janeiro: Embrapa Solos, 1999. 412 p.

FELFILI, J.M. Structure and dynamics of a gallery forest in Central Brazil. 1993. (D.Phil. Thesis) - University of Oxford, Oxford, 1993.

FELFILI, J. M. et al. Projeto biogeografia do bioma cerrado: vegetação e solos. Cadernos de Geociências do IBGE, v. 12, p. 75-166, 1994.

FELFILI, J.M. Diameter and height distributions of a gallery forest community and some of its main species in central Brazil over a six-year period (1985-1991). Revista Brasileira de Botânica, v. 20, p. 155-162, 1997.

FELFILI, J.M. Determinação de padrões de distribuição de espécies em uma mata de galeria no Brasil Central com a utilização de técnica de análise multivariada. Boletim do Herbário Ezechias Paulo Heringer, v. 2, p. 35- 48, 1998.

FELFILI, J.M. et al. O projeto biogeografia do bioma cerrado: hipóteses e padronização da metodologia. In: GARAY, I.; DIAS, B. (Ed.). Conservação da biodiversidade em ecossistemas tropicais. Petrópolis, RJ: [s.n.], 2001a p.157-173.

FELFILI, J.M. et al. Flora fanerogâmica das matas de galeria e ciliares do Brasil Central. In: RIBEIRO, J.F.; FONSECA, C.E.L.; SOUZA-SILVA, J.C. (Ed.) Cerrado caracterização e recuperação de matas de galeria. Planaltina, DF: [s.n.], 2001b v. 1, p. 195-209.

HARIDASAN, M. et al. Gradient analysis of soil properties and phytosociological parameters of some gallery forests of the "Chapada dos Veadeiros”. In: ENCINA, J.I.; KLEINN, C.

Proceedings of the International Symposium on Assessment and Monitoring of Forests in Tropical Dry Regions with Special Reference to Gallery Forests. Brasília: EDUNB, 1997. p. 259 - 276. 
HARPER, J.L. Population biology of plants. London: Academic Press, 1990. 892 p.

LEPS, J.; STURSA, J. Species-area curve, life history strategies, and succession: a field test of relationships. Vegetatio, v. 83, p. 249-257, 1989.

MEYER. H. A. Structure, growth and drain in balanced uneven-aged forest. Journal of Forestry, v. 50, p. 85-92, 1952.

MUELlER-DOMBOIS, D.; ELLENBERG, H. Aims and methods of vegetation ecology. New York: J. Willey \& Sons, 1974.

NÓBREGA, M.G.G. Fitossociologia e comunidades na mata de galeria Cabeça-de-Veado, no Jardim Botânico de Brasília, Brasília, DF. $199967 \mathrm{f}$. Dissertação (Mestrado em Ciência Florestal) Universidade de Brasília, Brasília, 1999.

OLIVEIRA-FILHO, A.T. et al. Effect of flooding regime and understorey bamboos in the physiognomy and tree species composition of a tropical semideciduous forest in Southeastern Brazil. Vegetatio, v. 113, p. 99-124, 1994.

OLIVEIRA-FILHO, A.T.; RATTER, J.A. A study of the origin of Central Brazilian forests by the analysis of plant species distribution patterns. Edinburgh Journal of Botany, v. 52, p. 141-194. 1995.

PEREIRA, B.A.S. et al. Reserva ecológica do IBGE (Brasília, DF): aspectos históricos e fisiográficos. Boletim da FBCN, v. 24, p. 30-43, 1989.

PEREIRA, B.A.S.; SILVA, M.A.; MENDONÇA, R.C. Reserva ecológica do IBGE (Brasília, DF): lista das plantas vasculares. Brasília: IBGE,1993.

PIELOU, E. C. Ecological diversity. New York: John Wiley \& Sons, 1975.

REZENDE, A.V. et al. Comparison of Gallery Forests on Well-Drained Soils in Veadeiros Plateaux, Goiás, Brazil. In: ENCINA, J.I.; KLEINN,

C. Proceedings of the International Symposium on Assessment and Monitoring of Forests in Tropical Dry Regions With Special Reference to Gallery Forests. Brasília: EDUNB, 1997. p. 365-378.
RIBEIRO, J. F.; WALTER, B. M. T. Fitofisionomias do bioma cerrado. Cerrado: ambiente e flora. [S.l.: s.n.], 1998. p. 89-169.

SILVA JÚNIOR, M.C. Tree communities of the gallery forest of the IBGE Ecological Reserve, Federal District, Brazil. 257f. (D.Phil. Thesis) - University of Edinburgh, Edinburgh, 1995.

SILVA JÚNIOR, M.C.; FURLEY, P.A.; RATTER, J.A. Variations in tree communities and soils with slope in Gallery Forest, Federal District, Brazil. In: ANDERSON, M. G; BROOKS, S. M. Advances in hill slope processes, v.1, n.20, p. 451469, 1996.

SILVA JÚNIOR, M.C. Relationships between the tree communities of the Pitoco, Monjolo and Taquara Gallery Forests and Environmental Factors. In: ENCINA, J.I.; KLEINN, C.

International Symposium on Assessment and Monitoring of Forests in Tropical Dry Regions with Special Reference to Gallery Forests, Brasília. Proceedings... EDUNB, 1997. p. 287-298.

SILVA JÚNIOR, M.C. Comunidades de árvores e sua relação com os solos na Mata do Pitoco, Reserva Ecológica do IBGE, Brasília-DF. Revista Árvore, v. 22, p. 29 - 40, 1998.

SILVA JÚNIOR, M.C. et al. Análise florística de matas de galeria no Distrito Federal. In: RIBEIRO, J. F. Cerrado - Matas de Galeria. Planaltina, DF: EMBRAPA-CPAC, 1998. p. 52-84.

SILVA JÚNIOR, M.C. Composição florística, fitossociologia e estrutura diamétrica na mata de galeria do Monjolo, Reserva Ecológica do IBGE, DF. Boletim do Herbário Ezechias Paulo Heringer, v. 4, p. 30-45, 1999.

SILVA JÚNIOR, M.C. Comparação entre matas de galeria no Distrito Federal e a efetividade do código florestal na proteção de sua diversidade arbórea. Acta Botanica Brasílica, v. 15, n. 1, p. 111-118, 2001.

R. Árvore, Viçosa-MG, v.28, n.3, p. 419-428, 2004 
SILVA JÚNIOR, M.C. et al. Análise da flora arbórea de Matas de Galeria no Distrito Federal: 21 levantamentos. In: RIBEIRO, J.F.; FONSECA, C.E.L.; SOUSA-SILVA, J.C. Cerrado:

caracterização e recuperação de Matas de Galeria. Planaltina, DF: EMBRAPA Cerrados, 2001. p. 142-191.

SPIEGEL, M.P. Estatística. São Paulo: McGrawHill, 1976.

STEBBINGS, G.L. Flowering plants. Evolution above the species level. Cambridge: [s.n.], 1974. 397 p.
UNESCO. Vegetação no Distrito Federal: tempo e espaço. Brasília: 2003. 74 p.

WALTER, B. M. T.; RIBEIRO, J. F. Spatial floristic pattern in gallery forest in the cerrado region, Brazil. In: ENCINA, J.I.; KLEINN, C. In: INTERNATIONAL SYMPOSIUM ON ASSESSMENT AND MONITORING OF FORESTS IN TROPICAL DRY REGIONS WITH SPECIAL REFERENCE TO GALLERY FORESTS, 1997, Brasília: Proceedings... Brasília: EDUNB, 1997. p. 339-349. 